\title{
HIV-1 protease inhibitors: effects on HIV-2 replication and resistance
}

\author{
Luis Menéndez-Arias ${ }^{1}$ and József Tözsér ${ }^{2}$
}

${ }^{1}$ Centro de Biología Molecular “Severo Ochoa”, Consejo Superior de Investigaciones Científicas Universidad Autónoma de Madrid, Cantoblanco, 28049 Madrid, Spain

${ }^{2}$ Department of Biochemistry and Molecular Biology, Faculty of Medicine, University of Debrecen, Debrecen, H-4012 Hungary

Corresponding author: Menéndez-Arias, L. (1menendez@cbm.uam.es) 
Novel antiretroviral drugs include protease (PR) inhibitors (e.g., atazanavir, tipranavir and darunavir) that block human immunodeficiency virus type 1 (HIV-1) maturation while showing remarkable antiviral potency on drug-resistant isolates. However, the strains used as prototypes in the design of the novel drugs belong to a specific clade (i.e. HIV-1 - group M - subtype B), which is the most prevalent in developed countries. At the same time, there is an increasing concern on the expansion of other HIV-1 clades as well as other related retroviruses such as HIV-2. The HIV-2 PR is weakly inhibited by some PR inhibitors (e.g. amprenavir), and little is known on the mutational pathways leading to drug resistance in this virus. The design of specific PR inhibitors targeting HIV-2, or potent drugs showing broad specificity on HIV-1 and HIV-2 clades remain as major challenges for the future.

\section{Introduction}

Since its first report in 1981, the acquired immunodeficiency syndrome (AIDS) has been continuously spreading. As of December 2006, there were around 40 million people infected with the human immunodeficiency virus (HIV) in the World. There are two main types of HIV, type 1 (HIV-1) and type 2 (HIV-2), with HIV-1 being the most prevalent in the worldwide pandemic. The HIV-2 is mainly present in West Africa, where it was discovered in 1986 [1], and infects around one million people worldwide. HIV-2 is slowly but continuously spreading to Europe, Asia and the Americas, reaching a significant prevalence in countries such as Portugal or India. In Portugal, HIV-2 accounts for approximately $4 \%$ of the total number of AIDS cases (information available at http://www.insarj.pt). 
Compared with HIV-1, HIV-2 is less transmissible and less pathogenic, with lower viral loads while asymptomatic and a slower progression to AIDS [2]. However, cohort studies and clinical trials are difficult to conduct due to the smaller number of people infected with HIV-2. In addition, monitoring the infection is complicated by the lack of a commercially available assay for measurement of plasma HIV-2 viremia. Currently approved antiretroviral drugs have been designed against HIV-1 (its viral life cycle is shown in Figure 1), and our knowledge on the efficiency of those compounds on HIV-2, both in vivo and in vitro, is still incomplete. The mutational patterns involved in the acquisition of drug resistance in HIV-2 strains may be different from those observed in HIV-1. In some cases, the comparison of HIV-1 and HIV-2 genotypes reveals the existence of amino acid sequence differences that have been associated with drug resistance in HIV-1. These differences are responsible in part for the low accuracy of genotypic interpretation algorithms developed for HIV-1, in predicting HIV-2 drug resistance. On the other hand, recombinant virus-based susceptibility assays use HIV-1 group M subtype B-based vectors into which patient-derived protease (PR) and/or reverse transcriptase (RT) sequences are transferred [3].

HIV-2 shows natural resistance to the three licensed non-nucleoside RT inhibitors (i.e., nevirapine, delavirdine and efavirenz). In contrast, nucleoside RT inhibitors such as zidovudine, lamivudine, stavudine, zalcitabine and abacavir, as well as the acyclic phosphonate tenofovir retain full activity against HIV-2 strains in phenotypic assays [4]. Since the introduction of potent antiretroviral drug combination therapies, PR inhibitors have contributed to the significant reduction of morbidity and mortality due to HIV-1 infection in developed countries [5]. However, the efficacy of PR inhibitors on HIV-2 has been poorly documented.

In this review, we will discuss on the differences between the inhibitor binding site of HIV-1 and HIV-2 PRs and interactions involved in binding currently approved PR inhibitors, 
emphasizing on the specific mutational patterns appearing in vitro and in vivo during HIV-2 infections.

\section{Comparison of the HIV-1 and HIV-2 PRs}

HIV PRs are homodimeric enzymes composed of two polypeptide chains of 99 residues. HIV1 and HIV-2 PRs share 39-48 \% identical amino acid sequences depending on the strain of virus being compared. For example, the PR of the strain HIV-1 $1_{\mathrm{HXB} 2}$ contains 48 amino acid residues which are conserved in the strain HIV-2 $2_{\text {ROD }}$ (Figure 2). The first detailed comparison of the HIV-1 and HIV-2 PR sequences and structures was made by Gustchina and Weber [6]. A model structure of the HIV-2 PR was then generated based on the crystal structure of the HIV-1 PR complexed with an inhibitor. As of today, there are more than two hundred crystal (or NMR) structures of wild-type and mutant HIV-1 PRs deposited in the Protein Data Bank (PDB). However, the number of HIV-2 PR structures is considerably smaller (less than 30, information is available at http://www.pdb.org). Most of the HIV-2 PR structures were obtained with inhibitors that have not been used in AIDS therapy. Indinavir is the only approved inhibitor whose structure has been solved in a complex with HIV-2 PR (PDB code 1HSH) (Figure 3). Despite the limited information on HIV-2 PRs, it is clear that the overall topology of this enzyme is very similar to that described for the HIV-1 PR.

HIV PR homodimers contain conserved Asp residues at position 25 of each unit. These Asp residues are part of the active site, which is held in a rigid position by a network of hydrogen bonds, known as the "fireman's grip". In the absence of ligand, both PRs show perfect symmetry. Each subunit contains two layers of $\beta$ sheets whose strands are oriented in an orthogonal fashion. These structures provide a hydrophobic core. In addition to the fireman's grip supporting the catalytic site, both subunits contain a flexible surface $\beta$-hairpin, known as the "flap" region (residues 42-58). These structures are flexible in the absence of the 
ligand, and close down upon binding of the inhibitor or the substrate (Figure 3). A fourstranded $\beta$ sheet formed by the $\mathrm{N}$ - and C-termini (residues 1-5 and 95-99) of each subunit provides a series of hydrogen bonds that appear to be critical for dimerization.

As originally suggested [6], all of the important structural elements are highly conserved, especially around the catalytic Asp residues (positions 23-30), as well as at a short region including residues $86-88$ which anchor the catalytic amino acids at an appropriate conformation for catalysis. The highly conserved Arg-87, which resides on the $\alpha$-helix comprising residues 87-91, forms a hydrogen bond with the side-chain of Asp-29. This interaction plays a critical role in HIV-1 PR dimerization [7].

HIV-1 reverse transcriptase is an error-prone enzyme (reviewed in [8]). Error rates of $10^{-4}$ mutations per nucleotide per cycle have been reported for HIV-1. These values are consistent with the extensive sequence variability observed in HIV populations. As for HIV-1 PR, studies on the variability of the HIV-2 PR-coding region have shown that sequence variation occurs mainly at surface positions, while the structurally important regions remain conserved [9-14] (Figure 2). The degree of PR sequence conservation ( $<1 \%$ variability) in HIV-2 isolates obtained from untreated patients is around $56 \%$, somewhat lower than for HIV1 PRs that shows conservation in 68 out of 99 residues [15]. In addition, there are substantial differences in the location of polymorphic sites. Thus, many of the polymorphic sites of HIV-2 PR (e.g., at positions 17, 40, 46, 68, 79 and 91) are highly conserved in the HIV-1 PR, and conversely, many of the highly polymorphic sites in the HIV-1 PR [9] are highly conserved in the HIV-2 PR (e.g., at positions 13, 37, 63, 64, 69 and 93).

The substrate binding pockets of HIV-1 and HIV-2 PRs are formed by residues 8, 23, $25,27-30,32,47-50,53,76,80-82$ and 84 . A schematic diagram of the substrate-binding site of HIV-2 PR showing the modelled interactions of the bound substrate representing the MA $\downarrow$ CA cleavage site is given in Figure 4. The substrate binds to either HIV-1 or HIV-2 PRs in an 
extended $\beta$ conformation and it is anchored by several hydrogen bonds. Substrate-based inhibitors bind in a very similar manner. The enzyme recognizes at least seven substrate residues, from P4 to P3' (Figure 4). Although these binding sites have been individually characterized based on detailed cleavage specificity studies, as well as HIV PR-inhibitor crystal structures, interactions defining PR specificity appear to show very strong sequence context dependence (reviewed in [16]). Importantly, there are only four residues involved in ligand binding which are different between HIV-1 and HIV-2 PRs. These residues are all involved in conservative substitutions that represent a loss or gain of methyl groups. Thus, Val-32, Ile-47, Leu-76 and Val-82 in HIV-1 PR change to Ile-32, Val-47, Met-76 and Ile-82 in the HIV-2 PR, respectively (Figures 2 and 4). Detailed analysis of the PR cleavage specificity, using oligopeptide substrates containing single-amino-acid substitutions in a substrate mimicking an HIV-1 Gag cleavage site combined with modelling studies, have revealed the importance of these residues to explain differences in substrate specificity between HIV-1 and HIV-2 PRs $[17,18]$.

\section{Clinically approved HIV-1 PR inhibitors that are also utilized in anti-HIV-2 therapy}

Currently there are nine clinically approved inhibitors developed against HIV-1 PR. These drugs are saquinavir, ritonavir, indinavir, amprenavir (also licensed as fosamprenavir), nelfinavir, lopinavir, atazanavir, tipranavir and darunavir (Figure 5). All of them except tipranavir, are compounds whose molecular structure mimics the structure of the PR substrates. Interactions between the PR and those inhibitors are mainly hydrophobic, and therefore, similar to those involved in enzyme-substrate interactions. Typically, PR inhibitors contain a phenyl residue at $\mathrm{P} 1$ or $\mathrm{P} 1$ ' positions. Another common feature of most PR inhibitors is that they contain a nonhydrolyzable transition-state mimic (e.g., a hydoxyethylamine group at the site corresponding to the cleavable bond in the substrates). Saquinavir was the first HIV-1 PR 
inhibitor that was licensed for therapy. Its chemical structure resembles an HIV-1 cleavage site (see Figure 5), where the Pro residue at position P1' was replaced by a saturated isoquinoline ring. Indinavir and nelfinavir also mimic the characteristic $\operatorname{Tyr}(\mathrm{Phe}) \downarrow$ Pro cleavage site.

Ritonavir was developed from a symmetric molecule, while amprenavir is a sulfonamide compound. Lopinavir was the first second-generation PR inhibitor. Its core is identical to that of ritonavir, and it was designed to diminish the interactions of the inhibitor with Val-82, a residue that is frequently mutated in HIV-1 drug-resistant strains. New, second-generation inhibitors, such as atazanavir or darunavir, have been designed to combat drug-resistant mutants mainly by increasing the number of polar interactions with main chain atoms of the PR [19]. At present, ritonavir is used only at low doses, to improve the pharmacokinetic properties of coadministered PR inhibitors.

\section{Molecular basis of HIV-2 PR drug resistance}

The molecular basis of HIV-1 drug resistance have been studied extensively. An important conclusion obtained from the analysis of crystal structures and kinetic studies with HIV PRs was that most amino acid changes that confer drug resistance in HIV-1 involve mutations at positions that contact the inhibitor but not the substrates. Therefore, resistant enzymes are capable of catalysing the cleavages required for infection but are not inhibited by the drugs [20].

In contrast to HIV-1, very few data are available about the impact of primary or secondary PR mutations on both viral fitness and the level of drug resistance in HIV-2 infection. Nevertheless, based on the similar structure of the two enzymes, most of the knowledge obtained with HIV-1 can be utilized also in HIV-2 to predict the molecular effect of mutations observed in drug resistance. 
In addition to primary drug-resistance mutations involving residues of the HIV-1 PR ligand-binding pockets, which coincide with amino acid differences between HIV-1 and HIV-2 PRs (i.e., V32I, I47V, L76M and V82I), secondary mutations may interfere with inhibitor binding through indirect perturbations of the binding site, or by influencing the enzyme activity through changing their conformational flexibility or PR dimerization properties. The same effects are expected for HIV-2 PR. Therefore, the presence of L10I/V, G16E, K20V, L33V, E35G, M36I/V, M46I/V, Q58E, I62V, A71I/V, G73A and I93L in the HIV-2 PR sequence (Figure 2) could also have an important effect on drug resistance, since the same residues are selected in the HIV-1 PR under treatment with PR inhibitors $[21,22]$.

\section{HIV-2 susceptibility to PR inhibitors in cell culture assays}

Saquinavir, indinavir and ritonavir were found to be equally active against HIV-1 and the HIV2 strains ROD and EHO, in conventional MT-4/MTT susceptibility assays [4], although others have reported that ritonavir was less effective on HIV-2, in assays carried out with peripheral blood mononuclear cells (PBMCs) or MT-2 cells $[23,24]$. On the other hand, HIV-2 ROD and EHO isolates showed $\mathrm{IC}_{50}$ values for nelfinavir which were around 1.5 to 4 times higher than the ones obtained with reference HIV-1 strains $[4,23,24]$. The relatively weak inhibitory effect of nelfinavir (approx. 4.5-fold increase of the $\mathrm{IC}_{50}$ for the inhibitor) on HIV-2 has been recently confirmed in assays carried out with uninfected cord blood mononuclear cells, and strains CBL20, CBL-23 and MVP-15132 [13]. In this study, authors also showed that lopinavir was an effective inhibitor of HIV-2 strains, an observation in agreement with preliminary data reported by others $[25,26]$.

Natural resistance to amprenavir has been demonstrated for several HIV-2 strains (e.g. ROD, EHO) $[4,13,23-26]$. In phenotypic assays, HIV-2 strains showed $\mathrm{IC}_{50}$ s which were 8.8 to 30 times higher than those obtained with wild-type HIV-1 clones. 
Novel PR inhibitors, such as tipranavir, atazanavir or darunavir have been selected for their lack of cross-resistance with other PR inhibitors, but also for their higher specificity on the HIV-1 enzyme. Therefore, a loss of potency on HIV-2 strains would not be surprising. Although, in some cases, available information is still preliminary, HIV-2 strains appear to be resistant to atazanavir [25-27], but susceptible to darunavir [24,26]. HIV-2 resistance to tipranavir has been reported by several groups. Published $\mathrm{IC}_{50}$ values for this inhibitor were 2 to 9 times higher than those reported for HIV-1 strains $[13,25,26]$.

\section{Development of resistance in cell culture}

As indicated above, several amino acid residues of the wild-type HIV-2 PR are also selected under drug pressure in the HIV-1 PR [22]. However, unlike in the case of HIV-1, information regarding selection of drug-resistant HIV-2 strains in vitro is scarce. For several inhibitors, the mutational patterns involved in HIV-2 resistance are different from those observed with HIV-1. Thus, I82L develops quickly in HIV-2 strains under tipranavir resistance [13], while conferring high-level resistance to the inhibitor in phenotypic assays [13,28]. Cross-resistance between tipranavir and lopinavir due to the presence of Leu-82 was observed in phenotypic assays [13]. Unlike in the case of HIV-2, HIV-1 PR variants containing Leu-82 emerge as the result of secondary mutations in selection experiments carried out in the presence of tipranavir [29].

The substitution of Met for Ile-54 (I54M) has been observed in HIV-2 strains selected in vitro in the presence of amprenavir, nelfinavir, and indinavir [13]. In the case of indinavir, it develops quickly, while conferring high-level resistance to amprenavir, nelfinavir and lopinavir in phenotypic assays $[13,28]$. However, HIV-2 strains carrying the I54M substitution in their PR remained susceptible to saquinavir [28]. 
HIV-1 and HIV-2 show remarkable differences in their mutational pathways leading to nelfinavir resistance. While the emergence of D30N appears to be relevant for the acquisition of nelfinavir resistance in HIV-1 [30], this amino acid change was not observed in selection experiments carried out with HIV-2 strains. Instead, I82F alone or I54M alone or in combination with L90M or L99F were commonly identified in nelfinavir-resistant HIV-2 strains selected in cell culture [13]. I82F confers high-level resistance to indinavir and lopinavir in phenotypic assays, while combinations of I54M with other mutations such as I84V, L90M or L99F may confer resistance to several PR inhibitors such as amprenavir, indinavir, nelfinavir, tipranavir and lopinavir. The presence of L99F in combination with V62A has been observed in indinavir-resistant HIV-2 variants derived from the CBL-23 strain. The V62A/L99F combination confers high-level resistance to lopinavir and moderate resistance to nelfinavir and indinavir in phenotypic assays [13].

Lopinavir-resistant HIV-2 variants obtained after passage of the virus in the presence of the drug (up to $1 \mu \mathrm{M}$ ) contained the V47A substitution in the viral PR, often accompanied by D17N [31]. D17N appears to have a minor effect on resistance and fitness [31]. However, the V47A mutation, which is also frequently found in lopinavir-treated patients [28,32], decreases lopinavir susceptibility by $>10$-fold in phenotypic assays [28,31]. In contrast, V47A has a minor effect on ritonavir, nelfinavir, tipranavir and darunavir resistance. Isolates containing the V47A mutation were hypersusceptible to saquinavir [28,31]. For other inhibitors, conflicting results have been reported. Rodés et al. [28] have shown that V47A confers amprenavir and indinavir resistance, while having no effect on atazanavir susceptibility. In contrast, Masse et al. [31] found that amprenavir and indinavir susceptibilities were not influenced by the mutation, but atazanavir susceptibility was reduced by 10-fold when V47A was introduced in the sequence background of the HIV-2 ${ }_{\text {ROD }}$ strain. 
In HIV-1, I47V emerges as a major lopinavir resistance mutation [33], since two nucleotide changes are required to obtain the I47A substitution. However, I47A has been identified in HIV-1-infected patients demonstrating evolution of lopinavir resistance, although in combination with V32I (a characteristic mutation found in the HIV-2 PR) [34].

\section{Sequence polymorphisms and selection of mutations in HIV-2-infected patients treated with PR inhibitors}

In the HIV-1 PR, the substitution M46I has been identified as a relevant mutation in the acquisition of resistance to indinavir [35], ritonavir [36], nelfinavir [37] and lopinavir [34,38]. In the HIV-2 PR, 46I is predominant in drug-naïve individuals, where it has been found in $>89$ $\%$ of the isolates obtained from infected patients $[10,12,14]$. However, HIV-2 strains are susceptible to indinavir and ritonavir in vitro [4]. Although the efficacy of those drugs on HIV2 has been poorly documented, clinical studies involving a relatively large number of patients showed that there is a significant association between the emergence of mutation I82F and ritonavir or indinavir therapy [11,39]. Interestingly, the presence of L90M has been reported to appear in HIV-2 from patients receiving saquinavir, indinavir, ritonavir or nelfinavir, usually in combination with other relevant mutations (i.e. I54M, I54L, V71I, I82F or I84V) $[9,11,32,40,41]$.

In the case of nelfinavir, L90M appears to play a major role in the acquisition of resistance, as occurs with HIV-1 subtype C viruses [42], and unlike in the case of HIV-1 subtype B, where the combination D30N/N88S mediates the acquisition of high-level resistance to the inhibitor, in the absence of previous treatments with PR inhibitors [37].

In addition, a number of changes at positions rarely associated with HIV-1 drug resistance appear to be selected in HIV-2-infected patients treated with PR inhibitors. 
Examples are K7R, V62A and V62T, or L99F [9]. K7R appeared in patients treated with saquinavir, ritonavir or lopinavir and in those individuals, the substitution was associated with M46I but never with L90M. L99F has been found in patients failing treatment with ritonavir and nelfinavir, although in combination with S43I, K45R, I54M, V71I and A92T [43].

Although V47A has been identified as the major lopinavir resistance mutation both in vivo and in vitro $[28,31,32]$, the presence of $\mathrm{K} 45 \mathrm{R}$ and $\mathrm{I} 64 \mathrm{~V}$ in viral isolates from patients failing lopinavir treatment has been reported in two different studies $[28,42]$. The significance of those mutations, as well as others which are found less frequently in those patients (e.g. S19P, G48A, $\mathrm{I} 50 \mathrm{~V})$ is still uncertain.

\section{Conclusions}

The susceptibility of the HIV-2 PR to antiretroviral drugs appears to be determined by amino acid substitutions other than those contributing to antiretroviral drug resistance in HIV-1. As discussed above, sequence differences between the HIV-1 and HIV-2 PRs are expected to have a large impact on the efficiency of the antiviral treatments. While amprenavir shows poor efficiency on HIV-2, the lower activity of nelfinavir, tipranavir and atazanavir, facilitates the development of resistance to those inhibitors. Inhibitors developed as drugs targeting heavily mutated HIV-1 PRs, such as lopinavir or tipranavir, are probably less effective on the HIV-2 PR due to the different sequence background that in some cases, would facilitate the rapid development of resistance, due to the emergence of single-nucleotide mutations that confer high-level resistance to the inhibitor. Examples are V47A in the case of ritonavir-boosted lopinavir therapy $[28,31,32]$, or I82L in the case of treatment with ritonavir-boosted tipranavir [13]. In addition to the limited knowledge about how to treat and interpret results from genotypic resistance assays in HIV-2 infection, therapeutic options might be limited in the long term due to inefficacy of several PR inhibitors (i.e. poor inhibitory activity and easy 
development of resistance). In this scenario, the design and development of novel specific drugs targeting the HIV-2 PR will gain importance, particularly with an increasing prevalence of HIV-2 infections. Our current knowledge of the interactions between the HIV-1 PR and its inhibitors should provide a solid framework for the development of specific inhibitors of the HIV-2 PR through rational design. In this context, the available crystal structures of HIV-2 PR/inhibitor complexes should be an important aid to achieve that goal.

\section{Acknowledgements}

Grants from the Spanish-Hungarian Intergovernmental Science and Technology Cooperation Program (HH2005-0020), the Hungarian Science and Research Fund (OTKA K68288), and the Spanish Ministery of Health (Instituto de Salud Carlos III, RD06/0006/0025), as well as an institutional grant of Fundación Ramón Areces are acknowledged.

\section{References}

1 Clavel, F., et al. (1986) Isolation of a new retrovirus from West African patients with AIDS. Science 233, 343-346.

2 Bock, P.J. and Markovitz, D.M. (2001) Infection with HIV-2. AIDS 15 (Suppl. 5), S35S45.

3 Sebastian, S. and Faruki, H. (2004) Update on HIV resistance and resistance testing. Med. Res. Rev. 24, 115-125.

4 Witvrouw, M., et al. (2004) Susceptibility of HIV-2, SIV and SHIV to various anti-HIV-1 compounds: implications for treatment and postexposure prophylaxis. Antivir. Ther. 9, 5765. 
5 Palella, F.J., Jr., et al. (1998) Declining morbidity and mortality among patients with advanced human immunodeficiency virus infection. N. Engl. J. Med. 338, 853-860.

6 Gustchina, A. and Weber, I.T. (1991) Comparative analysis of the sequences and structures of HIV-1 and HIV-2 proteases. Proteins 10, 325-339.

7 Louis, J.M., et al. (2007) HIV-1 protease: Structure dynamics, and inhibition. $A d v$. Pharmacol. 55, 261-298.

8 Menéndez-Arias, L. (2002) Molecular basis of fidelity of DNA synthesis and nucleotide specificity of retroviral reverse transcriptases. Progr. Nucleic Acid Res. Mol. Biol. 71, 91147.

9 Colson, P., et al. (2004) Polymorphism and drug selected mutations in the protease gene of human immunodeficiency virus type 2 from patients living in Southern France. J. Clin. Microbiol. 42, 570-577.

10 Pieniazek, D., et al. (2004) HIV-2 protease sequences of subtypes A and B harbor multiple mutations associated with protease inhibitor resistance in HIV-1. AIDS 18, 495-502.

11 Damond, F., et al. (2005) Polymorphism of the human immunodeficiency virus type 2 (HIV-2) protease gene and selection of drug resistance mutations in HIV-2-infected patients treated with protease inhibitors. J. Clin. Microbiol. 43, 484-487.

12 Parreira, R., et al. (2006) Natural polymorphisms of HIV type 2 pol sequences from drug naive individuals. AIDS Res. Hum. Retroviruses 22, 1178-1182.

13 Ntemgwa, M., et al. (2007) Natural polymorphisms in the human immunodeficiency virus type 2 protease can accelerate time to development of resistance to protease inhibitors. Antimicrob. Agents Chemother. 51, 604-610.

14 Ruelle, J., et al. (2007) Genetic polymorphisms and resistance mutations of HIV type 2 in antiretroviral-naïve patients in Burkina Faso. AIDS Res. Hum. Retroviruses 23, 955-964. 
15 Ceccherini-Silberstein, F., et al. (2004) Identification of the minimal conserved structure of HIV-1 protease in the presence and absence of drug pressure. AIDS 18, F11-F19.

16 Tözsér, J. and Oroszlan, S. (2003) Proteolytic events of HIV-1 replication as targets for therapeutic intervention. Curr. Pharm. Des. 9, 1803-1815.

17 Tözsér, J., et al. (1991) Studies on the role of the S4 substrate binding site of HIV proteinases. FEBS Lett. 279, 356-360.

18 Tözsér, J., et al. (1992) Kinetic and modeling studies of S3-S3' subsites of HIV proteinases. Biochemistry 31, 4793-4800.

19 Ghosh, A.K., et al. (2006) Structure-based design of novel HIV-1 protease inhibitors to combat drug resistance. J. Med. Chem. 49, 5252-5261.

20 King, N.M., et al. (2004) Combating susceptibility to drug resistance: lessons from HIV-1 protease. Chem. Biol. 10, 1333-1338.

21 Menéndez-Arias, L. (2002) Targeting HIV: Antiretroviral therapy and development of drug resistance. Trends Pharmacol. Sci. 23, 381-388.

22 Johnson, V.A., et al. (2007) Update of the drug resistance mutations in HIV-1: 2007. Top. HIV Med. 15, 119-125.

23 Yoshimura, K., et al. (2002) A potent human immunodeficiency virus type 1 protease inhibitor, UIC-94003 (TMC-126), and selection of a novel (A28S) mutation in the protease active site. J. Virol. 76, 1349-1358.

24 Koh, Y., et al. (2003) Novel bis-tetrahydrofuranylurethane-containing nonpeptidic protease inhibitor (PI) UIC-94017 (TMC114) with potent activity against multi-PI-resistant human immunodeficiency virus in vitro. Antimicrob. Agents Chemother. 47, 3123-3129. 
25 Descamps, D., et al. (2006) In vitro phenotypic susceptibility of HIV-2 clinical isolates to protease inhibitors: amprenavir, atazanavir, lopinavir, and tipranavir. Antivir. Ther. 11, S103.

26 Desbois, D., et al. (2007) Phenotypic susceptibility in vitro to amprenavir, atazanavir, darunavir, lopinavir, and tipranavir of HIV-2 clinical isolates from the French ANRS HIV2 Cohort. $14^{\text {th }}$ Conference on Retroviruses and Opportunistic Infections, Los Angeles, CA, U.S.A., Abstract no. 615.

27 Gong, Y.-F., et al. (2006) Atazanavir susceptibility spectrum extends to HIV-1 non-B subtypes and HIV-2 viral isolates. Antivir. Ther. 11, S100.

28 Rodés, B., et al. (2006) Susceptibility to protease inhibitors in HIV-2 primary isolates from patients failing antiretroviral therapy. J. Antimicrob. Chemother. 57, 709-713.

29 Doyon, L., et al. (2005) Selection and characterization of HIV-1 showing reduced susceptibility to the non-peptidic protease inhibitor tipranavir. Antivir. Res. 68, 27-35.

30 Patick, A.K., et al. (1996) Antiviral and resistance studies of AG1343, an orally bioavailable inhibitor of human immunodeficiency virus protease. Antimicrob. Agents Chemother. 40, 292-297.

31 Masse, S., et al. (2007) In vitro selection and characterization of human immunodeficiency virus type 2 with decreased susceptibility to lopinavir. Antimicrob. Agents Chemother. 51, 3075-3080.

32 Rodés, B., et al. (2006) High rate of proV47A selection in HIV-2 patients failing lopinavirbased HAART. AIDS 20, 127-129.

33 Carrillo, A., et al. (1998) In vitro selection and characterization of human immunodeficiency virus type 1 variants with increased resistance to ABT-378, a novel protease inhibitor. J. Virol. 72, 7532-7241. 
34 Mo, H., et al. (2005) Selection of resistance in protease inhibitor-experienced, human immunodeficiency virus type 1-infected subjects failing lopinavir- and ritonavir-based therapy: mutation patterns and baseline correlates. J. Virol. 79, 3329-3338.

35 Condra, J.H., et al. (1996) Genetic correlates of in vivo viral resistance to indinavir, a human immunodeficiency virus type 1 protease inhibitor. J. Virol. 70, 8270-8276.

36 Molla, A., et al. (1996) Ordered accumulation of mutations in HIV protease confers resistance to ritonavir. Nat. Med. 2, 760-766.

37 Patick, A.K., et al. (1998) Genotypic and phenotypic characterization of human immunodeficiency virus type 1 variants isolated from patients treated with the protease inhibitor nelfinavir. Antimicrob. Agents Chemother. 42, 2637-2644.

38 Masquelier, B., et al. (2002) Human immunodeficiency virus type 1 genotypic and pharmacokinetic determinants of the virological response to lopinavir-ritonavir-containing therapy in protease inhibitor-experienced patients. Antimicrob. Agents Chemother. 46, 2926-2932.

39 Rodés, B., et al. (2000) Emergence of drug resistance mutations in human immunodeficiency virus type 2-infected subjects undergoing antiretroviral therapy. J. Clin. Microbiol. 38, 1370-1374.

40 Van der Ende, M.E., et al. (2003) Clinical, immunological and virological response to different antiretroviral regimens in a cohort of HIV-2-infected patients. AIDS 17 (suppl. 3), S55-S61.

41 Cavaco Silva, J., et al. (2007) Mutations in HIV-2 protease selected by protease-inhibitor therapy. Antivir. Ther. 12, S87.

42 Grossman, Z., et al. (2001) Genotypic variation of HIV-1 reverse transcriptase and protease: comparative analysis of clade C and clade B. AIDS 15, 1453-1460. 
43 Brandin, E., et al. (2003) pol gene sequence variation in Swedish HIV-2 patients failing antiretroviral therapy. AIDS Res. Hum. Retroviruses 19, 543-550.

\section{LEGENDS TO FIGURES}

Figure 1. Targets for therapeutic intervention in the HIV life cycle. Currently approved antiretroviral drugs block HIV infection at different steps of the viral life cycle: (i) virus entry through their interaction with gp120 or gp41(i.e. maraviroc and enfuvirtide, respectively); (ii) reverse transcription (i.e. RT inhibitors); (iii) integration (i.e. integrase (IN) inhibitors such as raltegravir), and (iv) maturation (i.e. PR inhibitors that block the conversion of immature virus into mature infectious virions).

Figure 2. Amino acid sequence comparison of HIV-1 and HIV-2 proteases of reference strains HXB2 (HIV-1) and ROD (HIV-2). Identical residues are marked with red asterisks. Conserved structurally important regions are highlighted on a grey background. Box A corresponds to the dimerization domain, box $\mathrm{B}$ to the active site region and the $\mathrm{C}$-terminal triad, and box $\mathrm{C}$ indicates the flap region. Amino acid differences between HIV-1 and HIV-2 PRs at positions relevant for resistance to HIV-1 PR inhibitors are indicated [22]. Major and minor PR inhibitor resistance mutations occur at the positions highlighted in yellow and light blue, respectively. Highly polymorphic positions ( $\geq 20 \%$ sequence variation) in the PR of HIV-2 subtype A isolates are indicated with blue letters. HIV-2 PR residues appearing with a frequency above 4 $\%$ are indicated below the reference sequence. The analysis of polymorphic sites in the HIV-2 PR was based on 164 published sequences of HIV-2 isolates obtained from naïve patients [914]. 
Figure 3. Crystallographic structures of human immunodeficiency virus proteases complexed with indinavir. In panels (A) and (B), the two polypeptide chains of the human immunodeficiency virus type 2 protease are represented as ribbon diagrams (red and green). The inhibitor is shown in yellow using a CPK model. The side chains chains of catalytic residues (Asp-25 in both subunits) are shown in blue. Atom coordinates were taken from PDB file 1HSH. Side and top views are shown in (A) and (B), respectively. (C) Crystal structure of human immunodeficiency virus type 1 protease complexed with indinavir (PDB file 1HSG).

Figure 4. Residues forming the substrate binding pocket of HIV proteases. Schematic representation of an oligopeptide (SQNY $\downarrow$ PIV) that mimicks the HIV-1 Gag MA $\downarrow$ CA cleavage site, showing its potential interactions with the substrate binding site of the HIV-2 protease. Primes indicate residues in the second subunit of the dimer. The relative size of each substrate is illustrated by the area enclosed by the curved line around each substrate side chain. Substrate binding residues that are commonly observed in HIV isolates with high-level resistance to one or more drugs are boxed, and the residue found in the wild-type HIV-1 is shown between parentheses. The amino acid residues interacting with ritonavir, lopinavir and darunavir (inhibitors shown in white) in the crystal structures of HIV-1 PR complexes are indicated below. Sequence positions of residues at less than $4 \AA$ from the inhibitor are given. Side chains and their corresponding amino acid are indicated for those residues located at less than $3.5 \AA$ of the inhibitor.

Figure 5. Inhibitors of human immunodeficiency virus type 1 (HIV-1) protease that are licensed to treat AIDS and AIDS-related malignancies. 

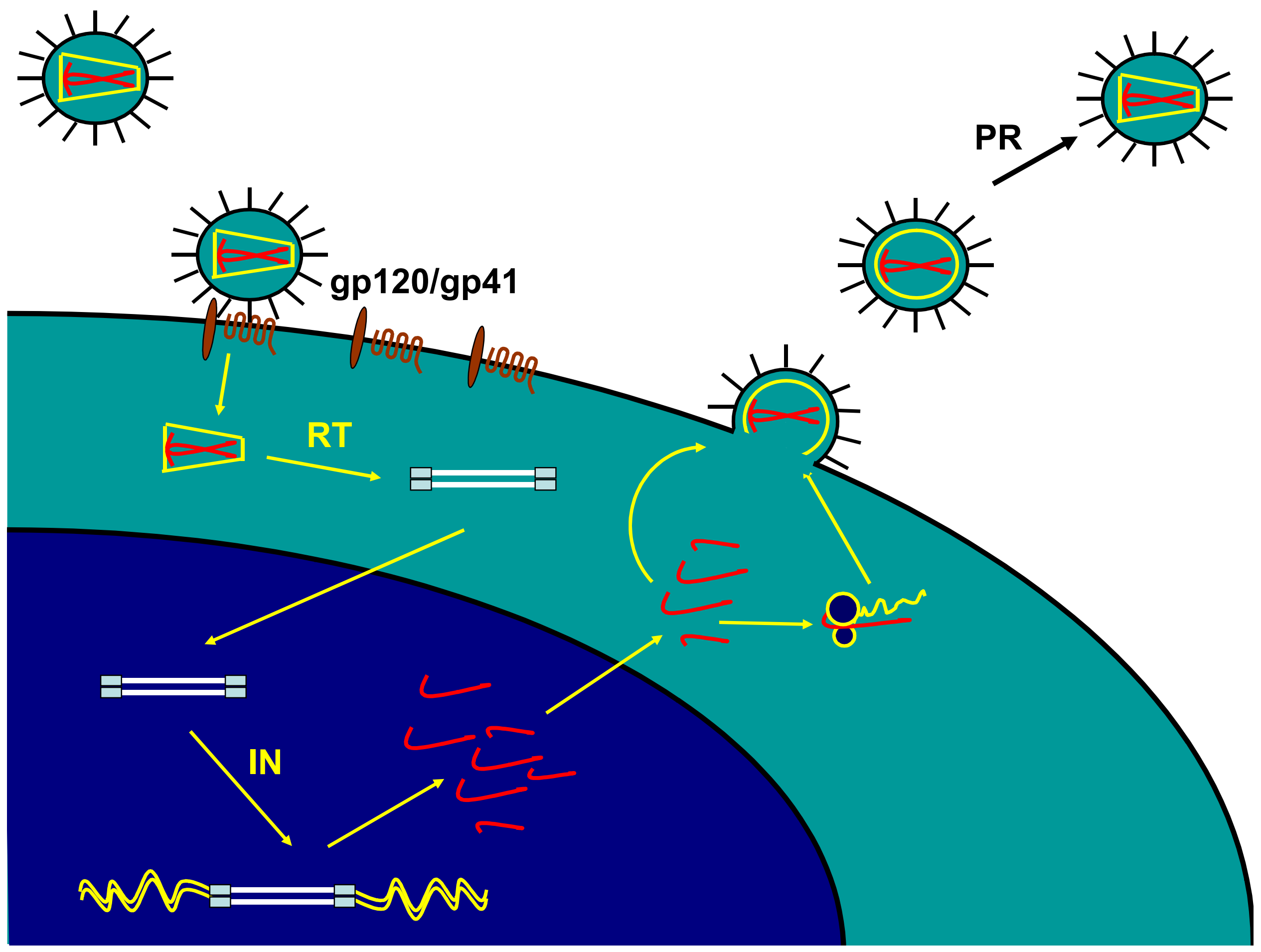


\begin{tabular}{|c|c|c|c|c|c|}
\hline & $A$ & & B & & C \\
\hline & & 20 & 30 & 40 & 50 \\
\hline HIV-1 & PQITLWQRPL & VTIKIGGQLK & $\begin{array}{l}\text { EALLDTGADD } \\
\text { A }\end{array}$ & $\underset{*}{\text { TVLEEMSLPG }}$ & RWKPKMIGGI \\
\hline \multirow[t]{4}{*}{ HIV-2 } & PQFSLWKRPV & VTAYIEGQPV & EVLLDTGADD & SIVAGIELGN & NYSPKIVGGI \\
\hline & & HV D & & $\begin{array}{l}\text { S } \\
\text { D }\end{array}$ & D T \\
\hline & C & & & $B$ & $A$ \\
\hline & 60 & 70 & 80 & 90 & 99 \\
\hline \multirow{2}{*}{ HIV-1 } & GGFIKVRQYD & QILIEICGHK & AIGTVLVGPT & PVNIIGRNLL & TQIGCTLNF \\
\hline & $* * * *$ & $* *$ & $* \quad * *$ & $* * * * * * *$ & $* * * *$ \\
\hline \multirow[t]{2}{*}{ HIV-2 } & GGFINTKEYK & NVEIEVLNKK & VRATIMTGDT & PINIFGRNIL & TALGMSLNL \\
\hline & & $\begin{array}{lll}\text { D } & K & \mathbf{R} \\
& \mathbf{R} & \mathbf{T}\end{array}$ & IK & v & \\
\hline
\end{tabular}

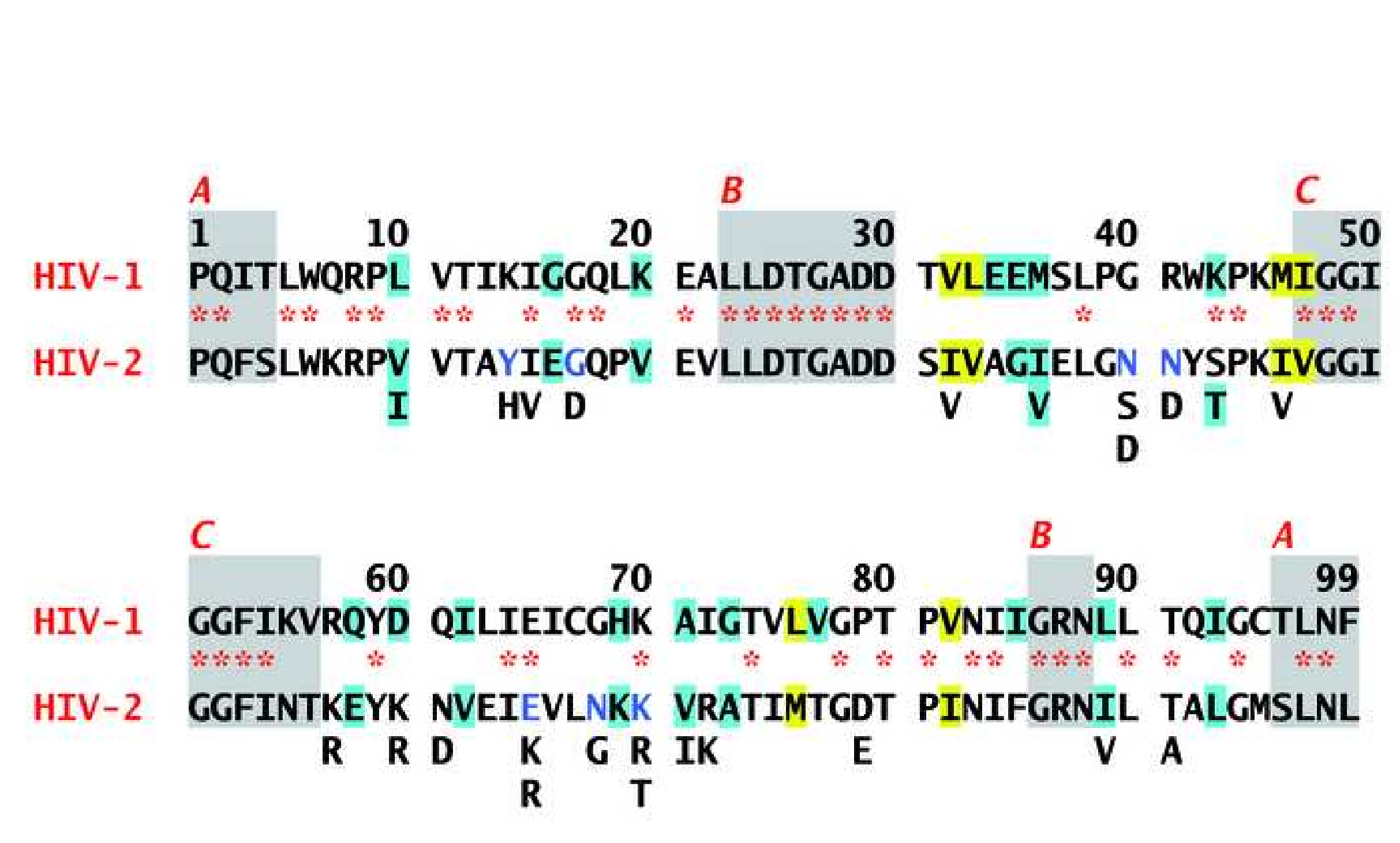

\section{c}

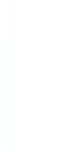

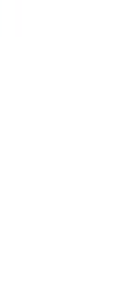

.

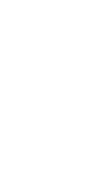

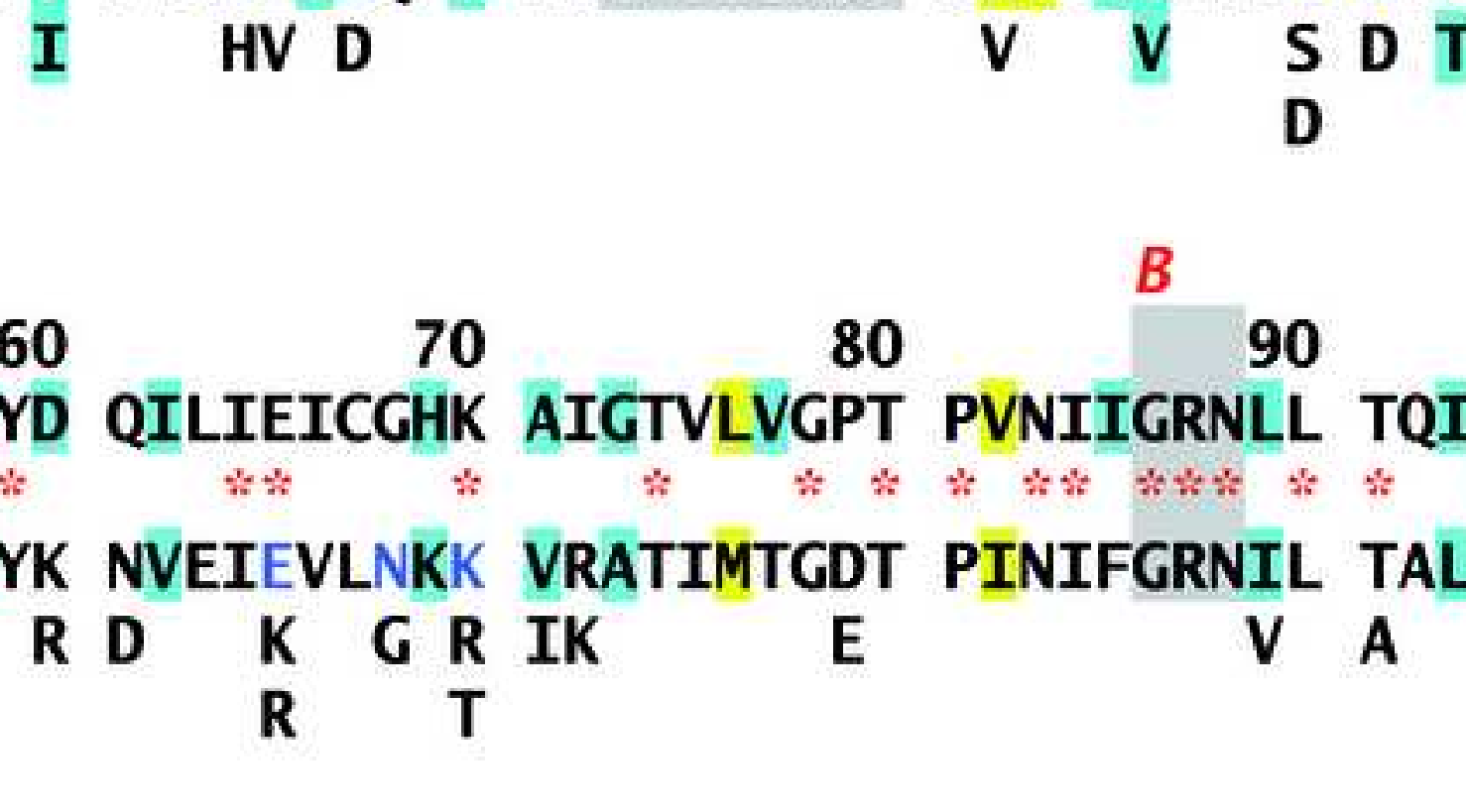

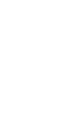

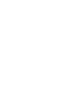
. . 


\section{Figure 4}

Click here to download high resolution image
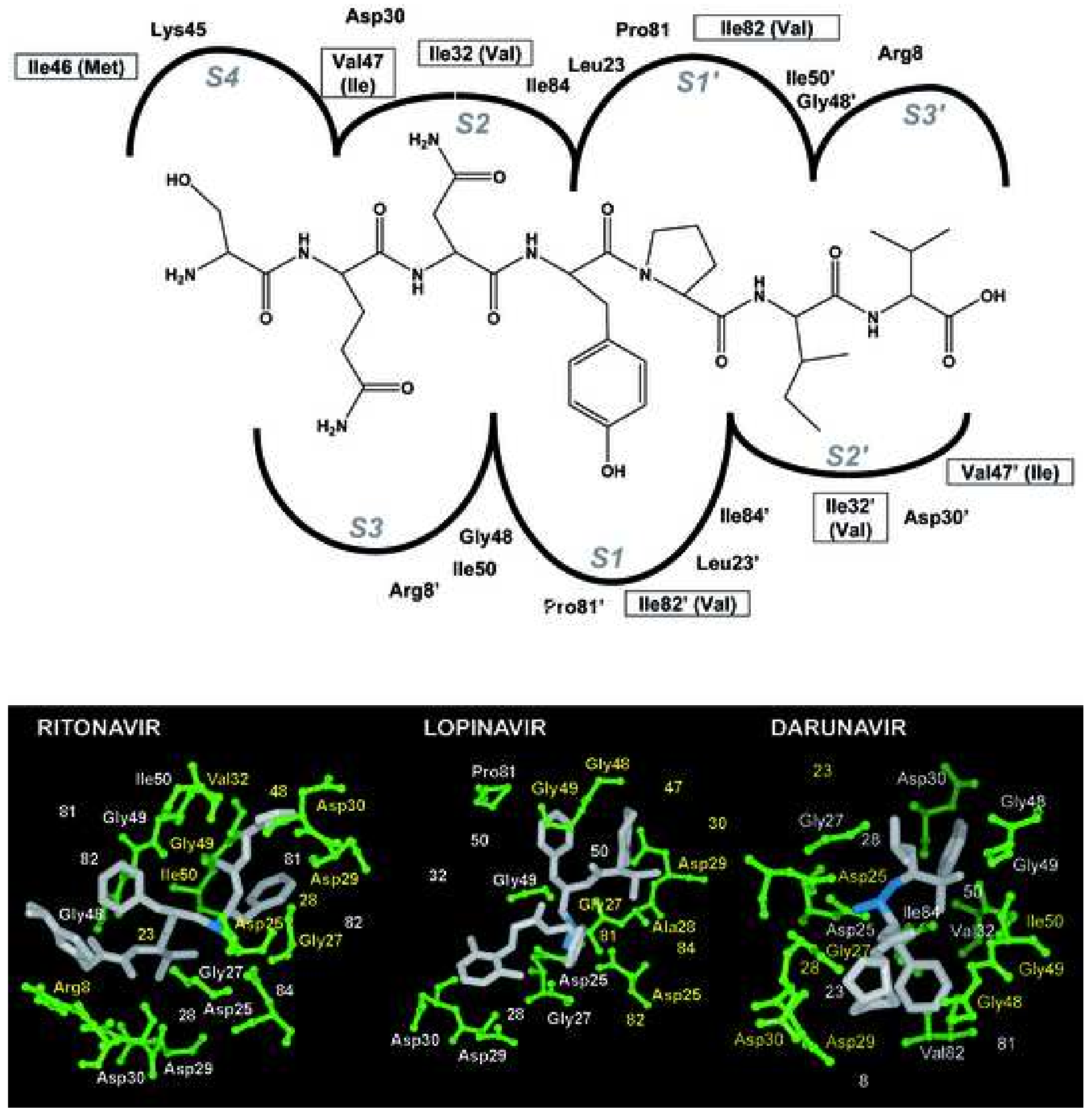
Figure 5

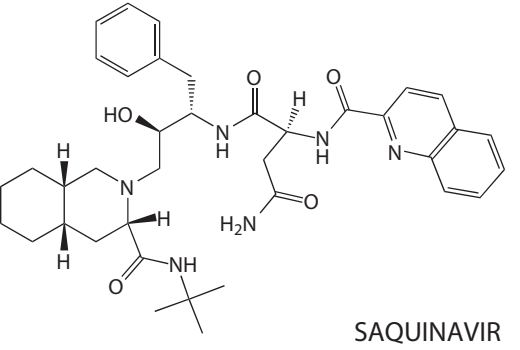

(c)

AMPRENAVIR

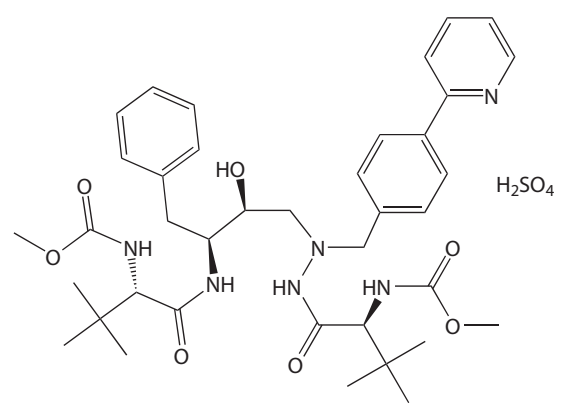

ATAZANAVIR

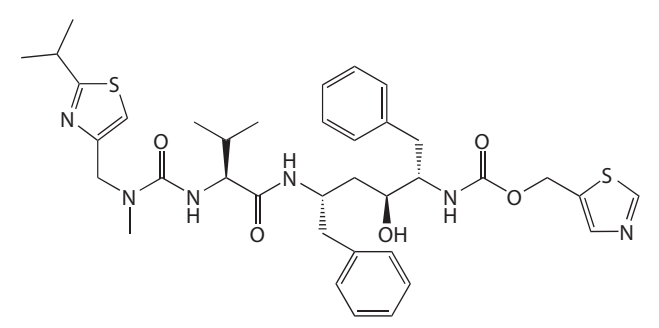

RITONAVIR

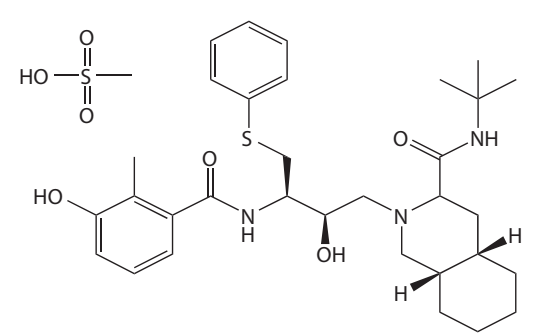

NELFINAVIR

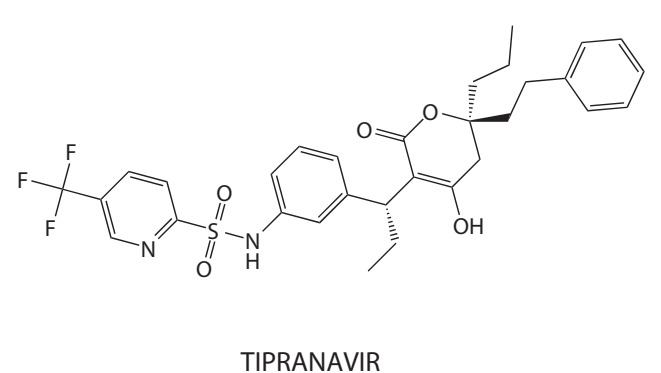

TIPRANAVIR

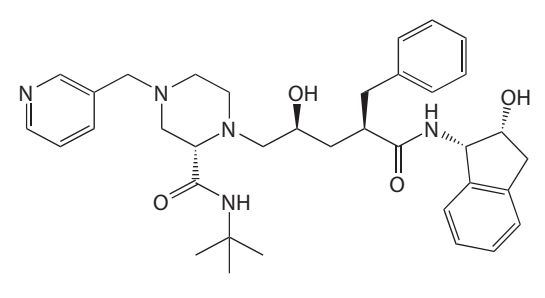

INDINAVIR

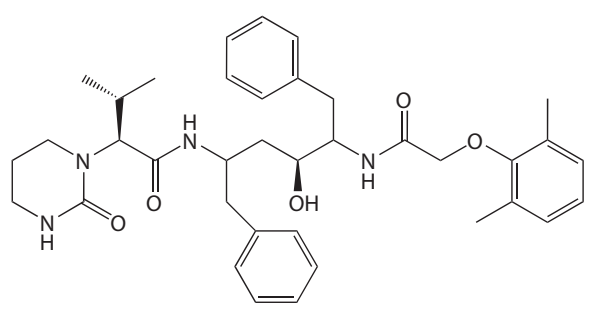

LOPINAVIR

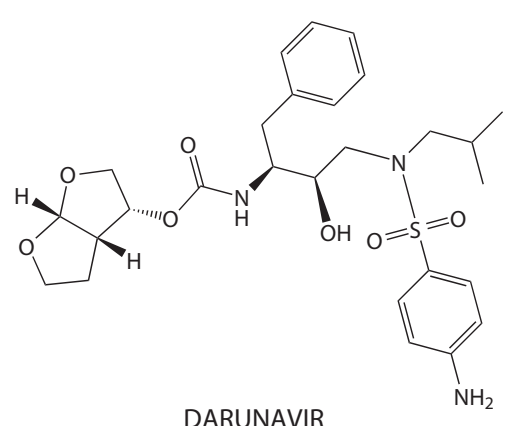




\section{${ }^{*}$ Cover letter with response to reviewers}
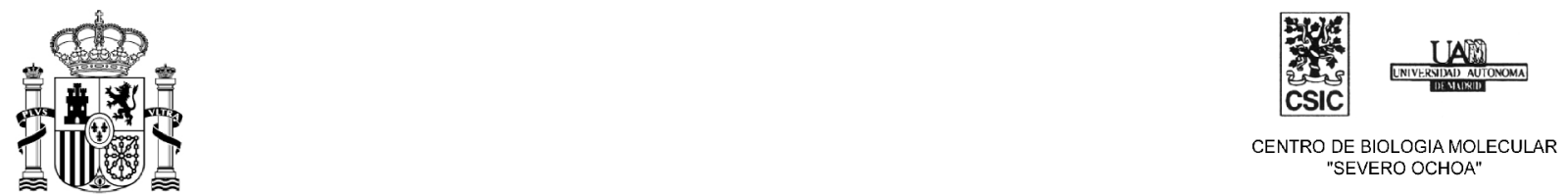

Lekshmy Balakrishnan, Ph.D.

Editor,

Trends in Pharmacological Sciences

Elsevier Ltd

84 Theobald's Road

London

UK WC1X 8RR

Ref.: TIPS-D-07-00047

Madrid, October 23rd, 2007

Dear Lekshmy,

Thank you very much for your e-mail of October $19^{\text {th }}$ concerning our manuscript entitled " $H I V-1$ protease inhibitors: effects on HIV-2 replication and resistance". We were happy to learn that both reviewers liked the paper and that it needed only minor revisions. We are pleased to send you a marked version of the revised manuscript, as well as the accompanying Figures.

In response to your comments and those of the reviewers', we have introduced the following changes in the revised manuscript:

\section{$\underline{\text { Editor's comments }}$}

1)... My only recommendation would be to include a figure in the 'Introduction' to show the various stages of HIV life cycle and which of these stages are targeted by RT and PR inhibitors...

Response: In the revised manuscript, we have introduced a new Figure (Fig. 1) indicating the relevant steps for drug intervention in the HIV life cycle. The figure is cited in the first paragraph of page 3.

\section{$\underline{\text { Reviewer no. } 1}$}

1) Unfortunately, limited data prevent the evaluation of all 9 approved PIs on HIV-2 both in vitro and in vivo. It would have been interesting, if the authors had focused a bit more on the effects of novel PIs, which are designed for drug resistant HIV-1 isolates and whether these may also possess activity against HIV-2 (e.g. darunavir, tipranavir).

Response: We agree with the reviewer on this point, but our perception is that the available information was not sufficient to make a strong issue on this matter. Please, note that references 25 to 27 , related to the point raised by the reviewer are abstracts from very recent conferences.

2) What research directions would the authors now propose for the development of PI therapy for HIV-2 infection? Is there a large enough market for product development?

Response: The question of market size is difficult to predict, since HIV infection keeps spreading, but at the same time, access to antiretroviral drugs will hopefully increase in the near future. There are some indications of a reduced prevalence of HIV-2 infections in Western African countries (Schim van der Loef et al., Int J Epidemiol 2006; 35: 1322-1328), but at this moment it is risky to speculate with this issue. About research directions, our suggestion would be to develop specific inhibitors of HIV-2 PR through rational design taking the available HIV-2 PR/inhibitor crystal structures as a reference for development. This is briefly discussed in the last 5 lines of the Conclusions section (page 13). 
3) One more need is that no commercially available viral load assay for the detection of HIV-2 now exists, making the monitoring of patients very difficult. The authors should add several sentences on this point.

Response: We have included this comment in the revised version of the manuscript at page 3, lines 4-5.

\section{$\underline{\text { Reviewer no. } 2}$}

1) Two of the figures need improvement. Figure 3: The lower panel appears to be low quality and the amino acid labels cannot be read.

Response: Figure 3 (now Figure 4) has been improved by increasing the size of amino acid labels in the lower left and lower center panels.

2) Figure 1: This figure is important and summarizes much information on resistant mutations and polymorphisms. There appears to be a small error at residue 71. Both V and I are highlighted in blue for HIV-2 protease. Only V should be highlighted, or else more explanation is required in the legend.

Response: It should be noted that some authors have considered A71I to be implicated in atazanavir resistance (Colonno et al. Antimicrob Agents Chemother 2003; 47: 1324-1333; Pellegrin et al. Antivir Ther 2006; 11: 421-429), and the mutation is found in the widely-used IAS mutation list (Johnson et al. Top HIV Med 2007; 15: 119-125). However, we agree with the reviewer that A71I is a secondary resistance mutation and that evidence for its implication in atazanavir resistance is not well-established. Therefore, the suggested correction has been made in Figure 1 (now Figure 2).

3) Minor typo: page 7, 2nd paragraph, line 1, "bases" should be "basis".

Response: The correction has been made (page 7, beginning of the second paragraph).

In addition, we have updated references 22 (Johnson et al., 2007) and 31 (Masse et al., 2007), and a new reference (no. 14; Ruelle et al., 2007) has been added to the bibliography. This reference corresponds to a paper published after the initial submission of our manuscript and affected the legend of the new Figure 2 by increasing the number of HIV-2 sequences considered in the analysis. Reference numbering has been modified accordingly. We have also rewritten the last paragraph of page 10 to clarify the differences between the results reported by Rodes et al. (2006) and Masse et al. (2007) on the effects of V47A.

Finally, we believe that we have satisfactorily responded to all the points addressed by the reviewers and this has resulted in an improved version of the manuscript. However, please let us know if additional modifications are necessary.

Thank you very much for your attention.

Regards,

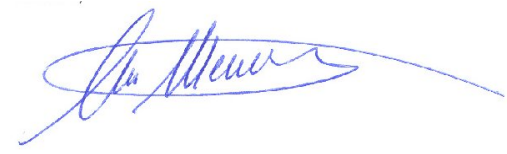

Luis Menéndez-Arias, Ph.D.

Jozsef Töszér, Ph.D. 
Luis Menéndez Arias, Ph.D.

Centro de Biología Molecular "Severo Ochoa"

CSIC-Universidad Autónoma de Madrid

c/Nicolás Cabrera, 1

Cantoblanco

28049 Madrid

Spain

Tel.: + 34911964494

Fax: + 34911964420

E-mail: 1menendez@cbm.uam.es

Jozsef Tözsér, Ph.D.

Laboratory of Retroviral Biochemistry

Department of Biochemistry and Molecular Biology

Medical and Health Science Center, Faculty of Medicine

Research Center for Molecular Medicine

UNIVERSITY OF DEBRECEN

Debrecen, Egyetem ter 1,

Life Science Building, 3.103

Postal Adress: H-4012 Debrecen, P.O.Box 6.

HUNGARY

Tel.: (+36-52) 411-600 x65314 (through operator)

(+36-52) 411-717 x65314 (through automatic switchboard)

Fax: $\quad(+36-52)$ 314-989

E-mail: tozser@indi.biochem.dote.hu 


\title{
HIV-1 protease inhibitors: effects on HIV-2 replication and resistance
}

\author{
Luis Menéndez-Arias ${ }^{1}$ and József Tözsér ${ }^{2}$
}

${ }^{1}$ Centro de Biología Molecular “Severo Ochoa”, Consejo Superior de Investigaciones Científicas Universidad Autónoma de Madrid, Cantoblanco, 28049 Madrid, Spain

${ }^{2}$ Department of Biochemistry and Molecular Biology, Faculty of Medicine, University of Debrecen, Debrecen, H-4012 Hungary

Corresponding author: Menéndez-Arias, L. (1menendez@cbm.uam.es) 
Novel antiretroviral drugs include protease (PR) inhibitors (e.g., atazanavir, tipranavir and darunavir) that block human immunodeficiency virus type 1 (HIV-1) maturation while showing remarkable antiviral potency on drug-resistant isolates. However, the strains used as prototypes in the design of the novel drugs belong to a specific clade (i.e. HIV-1 - group M - subtype B), which is the most prevalent in developed countries. At the same time, there is an increasing concern on the expansion of other HIV-1 clades as well as other related retroviruses such as HIV-2. The HIV-2 PR is weakly inhibited by some PR inhibitors (e.g. amprenavir), and little is known on the mutational pathways leading to drug resistance in this virus. The design of specific PR inhibitors targeting HIV-2, or potent drugs showing broad specificity on HIV-1 and HIV-2 clades remain as major challenges for the future.

\section{Introduction}

Since its first report in 1981, the acquired immunodeficiency syndrome (AIDS) has been continuously spreading. As of December 2006, there were around 40 million people infected with the human immunodeficiency virus (HIV) in the World. There are two main types of HIV, type 1 (HIV-1) and type 2 (HIV-2), with HIV-1 being the most prevalent in the worldwide pandemic. The HIV-2 is mainly present in West Africa, where it was discovered in 1986 [1], and infects around one million people worldwide. HIV-2 is slowly but continuously spreading to Europe, Asia and the Americas, reaching a significant prevalence in countries such as Portugal or India. In Portugal, HIV-2 accounts for approximately $4 \%$ of the total number of AIDS cases (information available at http://www.insarj.pt). 
Compared with HIV-1, HIV-2 is less transmissible and less pathogenic, with lower viral loads while asymptomatic and a slower progression to AIDS [2]. However, cohort studies and clinical trials are difficult to conduct due to the smaller number of people infected with HIV-2. In addition, monitoring the infection is complicated by the lack of a commercially available assay for measurement of plasma HIV-2 viremia. Currently approved antiretroviral drugs have been designed against HIV-1 (its viral life cycle is shown in Figure 1), and our knowledge on the efficiency of those compounds on HIV-2, both in vivo and in vitro, is still incomplete. The mutational patterns involved in the acquisition of drug resistance in HIV-2 strains may be different from those observed in HIV-1. In some cases, the comparison of HIV-1 and HIV-2 genotypes reveals the existence of amino acid sequence differences that have been associated with drug resistance in HIV-1. These differences are responsible in part for the low accuracy of genotypic interpretation algorithms developed for HIV-1, in predicting HIV-2 drug resistance. On the other hand, recombinant virus-based susceptibility assays use HIV-1 group M subtype B-based vectors into which patient-derived protease (PR) and/or reverse transcriptase (RT) sequences are transferred [3].

HIV-2 shows natural resistance to the three licensed non-nucleoside RT inhibitors (i.e., nevirapine, delavirdine and efavirenz). In contrast, nucleoside RT inhibitors such as zidovudine, lamivudine, stavudine, zalcitabine and abacavir, as well as the acyclic phosphonate tenofovir retain full activity against HIV-2 strains in phenotypic assays [4]. Since the introduction of potent antiretroviral drug combination therapies, PR inhibitors have contributed to the significant reduction of morbidity and mortality due to HIV-1 infection in developed countries [5]. However, the efficacy of PR inhibitors on HIV-2 has been poorly documented.

In this review, we will discuss on the differences between the inhibitor binding site of HIV-1 and HIV-2 PRs and interactions involved in binding currently approved PR inhibitors, 
emphasizing on the specific mutational patterns appearing in vitro and in vivo during HIV-2 infections.

\section{Comparison of the HIV-1 and HIV-2 PRs}

HIV PRs are homodimeric enzymes composed of two polypeptide chains of 99 residues. HIV1 and HIV-2 PRs share 39-48 \% identical amino acid sequences depending on the strain of virus being compared. For example, the PR of the strain HIV-1 $1_{\mathrm{HXB} 2}$ contains 48 amino acid residues which are conserved in the strain HIV-2 $2_{\text {ROD }}$ (Figure 2). The first detailed comparison of the HIV-1 and HIV-2 PR sequences and structures was made by Gustchina and Weber [6]. A model structure of the HIV-2 PR was then generated based on the crystal structure of the HIV-1 PR complexed with an inhibitor. As of today, there are more than two hundred crystal (or NMR) structures of wild-type and mutant HIV-1 PRs deposited in the Protein Data Bank (PDB). However, the number of HIV-2 PR structures is considerably smaller (less than 30, information is available at http://www.pdb.org). Most of the HIV-2 PR structures were obtained with inhibitors that have not been used in AIDS therapy. Indinavir is the only approved inhibitor whose structure has been solved in a complex with HIV-2 PR (PDB code 1HSH) (Figure 3). Despite the limited information on HIV-2 PRs, it is clear that the overall topology of this enzyme is very similar to that described for the HIV-1 PR.

HIV PR homodimers contain conserved Asp residues at position 25 of each unit. These Asp residues are part of the active site, which is held in a rigid position by a network of hydrogen bonds, known as the "fireman's grip". In the absence of ligand, both PRs show perfect symmetry. Each subunit contains two layers of $\beta$ sheets whose strands are oriented in an orthogonal fashion. These structures provide a hydrophobic core. In addition to the fireman's grip supporting the catalytic site, both subunits contain a flexible surface $\beta$-hairpin, known as the "flap" region (residues 42-58). These structures are flexible in the absence of the 
ligand, and close down upon binding of the inhibitor or the substrate (Figure 3). A fourstranded $\beta$ sheet formed by the $\mathrm{N}$ - and C-termini (residues 1-5 and 95-99) of each subunit provides a series of hydrogen bonds that appear to be critical for dimerization.

As originally suggested [6], all of the important structural elements are highly conserved, especially around the catalytic Asp residues (positions 23-30), as well as at a short region including residues $86-88$ which anchor the catalytic amino acids at an appropriate conformation for catalysis. The highly conserved Arg-87, which resides on the $\alpha$-helix comprising residues 87-91, forms a hydrogen bond with the side-chain of Asp-29. This interaction plays a critical role in HIV-1 PR dimerization [7].

HIV-1 reverse transcriptase is an error-prone enzyme (reviewed in [8]). Error rates of $10^{-4}$ mutations per nucleotide per cycle have been reported for HIV-1. These values are consistent with the extensive sequence variability observed in HIV populations. As for HIV-1 PR, studies on the variability of the HIV-2 PR-coding region have shown that sequence variation occurs mainly at surface positions, while the structurally important regions remain conserved [9-14] (Figure 2). The degree of PR sequence conservation ( $<1 \%$ variability) in HIV-2 isolates obtained from untreated patients is around $56 \%$, somewhat lower than for HIV1 PRs that shows conservation in 68 out of 99 residues [15]. In addition, there are substantial differences in the location of polymorphic sites. Thus, many of the polymorphic sites of HIV-2 PR (e.g., at positions 17, 40, 46, 68, 79 and 91) are highly conserved in the HIV-1 PR, and conversely, many of the highly polymorphic sites in the HIV-1 PR [9] are highly conserved in the HIV-2 PR (e.g., at positions 13, 37, 63, 64, 69 and 93).

The substrate binding pockets of HIV-1 and HIV-2 PRs are formed by residues 8, 23, $25,27-30,32,47-50,53,76,80-82$ and 84 . A schematic diagram of the substrate-binding site of HIV-2 PR showing the modelled interactions of the bound substrate representing the MA $\downarrow$ CA cleavage site is given in Figure 4. The substrate binds to either HIV-1 or HIV-2 PRs in an 
extended $\beta$ conformation and it is anchored by several hydrogen bonds. Substrate-based inhibitors bind in a very similar manner. The enzyme recognizes at least seven substrate residues, from P4 to P3' (Figure 4). Although these binding sites have been individually characterized based on detailed cleavage specificity studies, as well as HIV PR-inhibitor crystal structures, interactions defining PR specificity appear to show very strong sequence context dependence (reviewed in [16]). Importantly, there are only four residues involved in ligand binding which are different between HIV-1 and HIV-2 PRs. These residues are all involved in conservative substitutions that represent a loss or gain of methyl groups. Thus, Val-32, Ile-47, Leu-76 and Val-82 in HIV-1 PR change to Ile-32, Val-47, Met-76 and Ile-82 in the HIV-2 PR, respectively (Figures 2 and 4). Detailed analysis of the PR cleavage specificity, using oligopeptide substrates containing single-amino-acid substitutions in a substrate mimicking an HIV-1 Gag cleavage site combined with modelling studies, have revealed the importance of these residues to explain differences in substrate specificity between HIV-1 and HIV-2 PRs $[17,18]$.

\section{Clinically approved HIV-1 PR inhibitors that are also utilized in anti-HIV-2 therapy}

Currently there are nine clinically approved inhibitors developed against HIV-1 PR. These drugs are saquinavir, ritonavir, indinavir, amprenavir (also licensed as fosamprenavir), nelfinavir, lopinavir, atazanavir, tipranavir and darunavir (Figure 5). All of them except tipranavir, are compounds whose molecular structure mimics the structure of the PR substrates. Interactions between the PR and those inhibitors are mainly hydrophobic, and therefore, similar to those involved in enzyme-substrate interactions. Typically, PR inhibitors contain a phenyl residue at P1 or P1' positions. Another common feature of most PR inhibitors is that they contain a nonhydrolyzable transition-state mimic (e.g., a hydoxyethylamine group at the site corresponding to the cleavable bond in the substrates). Saquinavir was the first HIV-1 PR 
inhibitor that was licensed for therapy. Its chemical structure resembles an HIV-1 cleavage site (see Figure 5), where the Pro residue at position P1' was replaced by a saturated isoquinoline ring. Indinavir and nelfinavir also mimic the characteristic $\operatorname{Tyr}(\mathrm{Phe}) \downarrow$ Pro cleavage site.

Ritonavir was developed from a symmetric molecule, while amprenavir is a sulfonamide compound. Lopinavir was the first second-generation PR inhibitor. Its core is identical to that of ritonavir, and it was designed to diminish the interactions of the inhibitor with Val-82, a residue that is frequently mutated in HIV-1 drug-resistant strains. New, second-generation inhibitors, such as atazanavir or darunavir, have been designed to combat drug-resistant mutants mainly by increasing the number of polar interactions with main chain atoms of the PR [19]. At present, ritonavir is used only at low doses, to improve the pharmacokinetic properties of coadministered PR inhibitors.

\section{Molecular basis of HIV-2 PR drug resistance}

The molecular basis of HIV-1 drug resistance have been studied extensively. An important conclusion obtained from the analysis of crystal structures and kinetic studies with HIV PRs was that most amino acid changes that confer drug resistance in HIV-1 involve mutations at positions that contact the inhibitor but not the substrates. Therefore, resistant enzymes are capable of catalysing the cleavages required for infection but are not inhibited by the drugs [20].

In contrast to HIV-1, very few data are available about the impact of primary or secondary PR mutations on both viral fitness and the level of drug resistance in HIV-2 infection. Nevertheless, based on the similar structure of the two enzymes, most of the knowledge obtained with HIV-1 can be utilized also in HIV-2 to predict the molecular effect of mutations observed in drug resistance. 
In addition to primary drug-resistance mutations involving residues of the HIV-1 PR ligand-binding pockets, which coincide with amino acid differences between HIV-1 and HIV-2 PRs (i.e., V32I, I47V, L76M and V82I), secondary mutations may interfere with inhibitor binding through indirect perturbations of the binding site, or by influencing the enzyme activity through changing their conformational flexibility or PR dimerization properties. The same effects are expected for HIV-2 PR. Therefore, the presence of L10I/V, G16E, K20V, L33V, E35G, M36I/V, M46I/V, Q58E, I62V, A71I/V, G73A and I93L in the HIV-2 PR sequence (Figure 2) could also have an important effect on drug resistance, since the same residues are selected in the HIV-1 PR under treatment with PR inhibitors [21,22].

\section{HIV-2 susceptibility to PR inhibitors in cell culture assays}

Saquinavir, indinavir and ritonavir were found to be equally active against HIV-1 and the HIV2 strains ROD and EHO, in conventional MT-4/MTT susceptibility assays [4], although others have reported that ritonavir was less effective on HIV-2, in assays carried out with peripheral blood mononuclear cells (PBMCs) or MT-2 cells $[23,24]$. On the other hand, HIV-2 ROD and EHO isolates showed $\mathrm{IC}_{50}$ values for nelfinavir which were around 1.5 to 4 times higher than the ones obtained with reference HIV-1 strains $[4,23,24]$. The relatively weak inhibitory effect of nelfinavir (approx. 4.5-fold increase of the $\mathrm{IC}_{50}$ for the inhibitor) on HIV-2 has been recently confirmed in assays carried out with uninfected cord blood mononuclear cells, and strains CBL20, CBL-23 and MVP-15132 [13]. In this study, authors also showed that lopinavir was an effective inhibitor of HIV-2 strains, an observation in agreement with preliminary data reported by others $[25,26]$.

Natural resistance to amprenavir has been demonstrated for several HIV-2 strains (e.g. ROD, EHO) $[4,13,23-26]$. In phenotypic assays, HIV-2 strains showed $\mathrm{IC}_{50}$ s which were 8.8 to 30 times higher than those obtained with wild-type HIV-1 clones. 
Novel PR inhibitors, such as tipranavir, atazanavir or darunavir have been selected for their lack of cross-resistance with other PR inhibitors, but also for their higher specificity on the HIV-1 enzyme. Therefore, a loss of potency on HIV-2 strains would not be surprising. Although, in some cases, available information is still preliminary, HIV-2 strains appear to be resistant to atazanavir [25-27], but susceptible to darunavir [24,26]. HIV-2 resistance to tipranavir has been reported by several groups. Published $\mathrm{IC}_{50}$ values for this inhibitor were 2 to 9 times higher than those reported for HIV-1 strains $[13,25,26]$.

\section{Development of resistance in cell culture}

As indicated above, several amino acid residues of the wild-type HIV-2 PR are also selected under drug pressure in the HIV-1 PR [22]. However, unlike in the case of HIV-1, information regarding selection of drug-resistant HIV-2 strains in vitro is scarce. For several inhibitors, the mutational patterns involved in HIV-2 resistance are different from those observed with HIV-1. Thus, I82L develops quickly in HIV-2 strains under tipranavir resistance [13], while conferring high-level resistance to the inhibitor in phenotypic assays [13,28]. Cross-resistance between tipranavir and lopinavir due to the presence of Leu-82 was observed in phenotypic assays [13]. Unlike in the case of HIV-2, HIV-1 PR variants containing Leu-82 emerge as the result of secondary mutations in selection experiments carried out in the presence of tipranavir [29].

The substitution of Met for Ile-54 (I54M) has been observed in HIV-2 strains selected in vitro in the presence of amprenavir, nelfinavir, and indinavir [13]. In the case of indinavir, it develops quickly, while conferring high-level resistance to amprenavir, nelfinavir and lopinavir in phenotypic assays $[13,28]$. However, HIV-2 strains carrying the I54M substitution in their PR remained susceptible to saquinavir [28]. 
HIV-1 and HIV-2 show remarkable differences in their mutational pathways leading to nelfinavir resistance. While the emergence of D30N appears to be relevant for the acquisition of nelfinavir resistance in HIV-1 [30], this amino acid change was not observed in selection experiments carried out with HIV-2 strains. Instead, I82F alone or I54M alone or in combination with L90M or L99F were commonly identified in nelfinavir-resistant HIV-2 strains selected in cell culture [13]. I82F confers high-level resistance to indinavir and lopinavir in phenotypic assays, while combinations of I54M with other mutations such as I84V, L90M or L99F may confer resistance to several PR inhibitors such as amprenavir, indinavir, nelfinavir, tipranavir and lopinavir. The presence of L99F in combination with V62A has been observed in indinavir-resistant HIV-2 variants derived from the CBL-23 strain. The V62A/L99F combination confers high-level resistance to lopinavir and moderate resistance to nelfinavir and indinavir in phenotypic assays [13].

Lopinavir-resistant HIV-2 variants obtained after passage of the virus in the presence of the drug (up to $1 \mu \mathrm{M}$ ) contained the V47A substitution in the viral PR, often accompanied by D17N [31]. D17N appears to have a minor effect on resistance and fitness [31]. However, the V47A mutation, which is also frequently found in lopinavir-treated patients [28,32], decreases lopinavir susceptibility by $>10$-fold in phenotypic assays $[28,31]$. In contrast, V47A has a minor effect on ritonavir, nelfinavir, tipranavir and darunavir resistance. Isolates containing the V47A mutation were hypersusceptible to saquinavir [28,31]. For other inhibitors, conflicting results have been reported. Rodés et al. [28] have shown that V47A confers amprenavir and indinavir resistance, while having no effect on atazanavir susceptibility. In contrast, Masse et al. [31] found that amprenavir and indinavir susceptibilities were not influenced by the mutation, but atazanavir susceptibility was reduced by 10-fold when V47A was introduced in the sequence background of the HIV-2 ${ }_{\text {ROD }}$ strain. 
In HIV-1, I47V emerges as a major lopinavir resistance mutation [33], since two nucleotide changes are required to obtain the I47A substitution. However, I47A has been identified in HIV-1-infected patients demonstrating evolution of lopinavir resistance, although in combination with V32I (a characteristic mutation found in the HIV-2 PR) [34].

\section{Sequence polymorphisms and selection of mutations in HIV-2-infected patients treated with PR inhibitors}

In the HIV-1 PR, the substitution M46I has been identified as a relevant mutation in the acquisition of resistance to indinavir [35], ritonavir [36], nelfinavir [37] and lopinavir [34,38]. In the HIV-2 PR, 46I is predominant in drug-naïve individuals, where it has been found in $>89$ $\%$ of the isolates obtained from infected patients $[10,12,14]$. However, HIV-2 strains are susceptible to indinavir and ritonavir in vitro [4]. Although the efficacy of those drugs on HIV2 has been poorly documented, clinical studies involving a relatively large number of patients showed that there is a significant association between the emergence of mutation I82F and ritonavir or indinavir therapy [11,39]. Interestingly, the presence of L90M has been reported to appear in HIV-2 from patients receiving saquinavir, indinavir, ritonavir or nelfinavir, usually in combination with other relevant mutations (i.e. I54M, I54L, V71I, I82F or I84V) $[9,11,32,40,41]$.

In the case of nelfinavir, L90M appears to play a major role in the acquisition of resistance, as occurs with HIV-1 subtype C viruses [42], and unlike in the case of HIV-1 subtype B, where the combination D30N/N88S mediates the acquisition of high-level resistance to the inhibitor, in the absence of previous treatments with PR inhibitors [37].

In addition, a number of changes at positions rarely associated with HIV-1 drug resistance appear to be selected in HIV-2-infected patients treated with PR inhibitors. 
Examples are K7R, V62A and V62T, or L99F [9]. K7R appeared in patients treated with saquinavir, ritonavir or lopinavir and in those individuals, the substitution was associated with M46I but never with L90M. L99F has been found in patients failing treatment with ritonavir and nelfinavir, although in combination with S43I, K45R, I54M, V71I and A92T [43].

Although V47A has been identified as the major lopinavir resistance mutation both in vivo and in vitro $[28,31,32]$, the presence of $\mathrm{K} 45 \mathrm{R}$ and $\mathrm{I} 64 \mathrm{~V}$ in viral isolates from patients failing lopinavir treatment has been reported in two different studies $[28,42]$. The significance of those mutations, as well as others which are found less frequently in those patients (e.g. S19P, G48A, $\mathrm{I} 50 \mathrm{~V})$ is still uncertain.

\section{Conclusions}

The susceptibility of the HIV-2 PR to antiretroviral drugs appears to be determined by amino acid substitutions other than those contributing to antiretroviral drug resistance in HIV-1. As discussed above, sequence differences between the HIV-1 and HIV-2 PRs are expected to have a large impact on the efficiency of the antiviral treatments. While amprenavir shows poor efficiency on HIV-2, the lower activity of nelfinavir, tipranavir and atazanavir, facilitates the development of resistance to those inhibitors. Inhibitors developed as drugs targeting heavily mutated HIV-1 PRs, such as lopinavir or tipranavir, are probably less effective on the HIV-2 PR due to the different sequence background that in some cases, would facilitate the rapid development of resistance, due to the emergence of single-nucleotide mutations that confer high-level resistance to the inhibitor. Examples are V47A in the case of ritonavir-boosted lopinavir therapy $[28,31,32]$, or I82L in the case of treatment with ritonavir-boosted tipranavir [13]. In addition to the limited knowledge about how to treat and interpret results from genotypic resistance assays in HIV-2 infection, therapeutic options might be limited in the long term due to inefficacy of several PR inhibitors (i.e. poor inhibitory activity and easy 
development of resistance). In this scenario, the design and development of novel specific drugs targeting the HIV-2 PR will gain importance, particularly with an increasing prevalence of HIV-2 infections. Our current knowledge of the interactions between the HIV-1 PR and its inhibitors should provide a solid framework for the development of specific inhibitors of the HIV-2 PR through rational design. In this context, the available crystal structures of HIV-2 PR/inhibitor complexes should be an important aid to achieve that goal.

\section{Acknowledgements}

Grants from the Spanish-Hungarian Intergovernmental Science and Technology Cooperation Program (HH2005-0020), the Hungarian Science and Research Fund (OTKA K68288), and the Spanish Ministery of Health (Instituto de Salud Carlos III, RD06/0006/0025), as well as an institutional grant of Fundación Ramón Areces are acknowledged.

\section{References}

1 Clavel, F., et al. (1986) Isolation of a new retrovirus from West African patients with AIDS. Science 233, 343-346.

2 Bock, P.J. and Markovitz, D.M. (2001) Infection with HIV-2. AIDS 15 (Suppl. 5), S35S45.

3 Sebastian, S. and Faruki, H. (2004) Update on HIV resistance and resistance testing. Med. Res. Rev. 24, 115-125.

4 Witvrouw, M., et al. (2004) Susceptibility of HIV-2, SIV and SHIV to various anti-HIV-1 compounds: implications for treatment and postexposure prophylaxis. Antivir. Ther. 9, 5765. 
5 Palella, F.J., Jr., et al. (1998) Declining morbidity and mortality among patients with advanced human immunodeficiency virus infection. N. Engl. J. Med. 338, 853-860.

6 Gustchina, A. and Weber, I.T. (1991) Comparative analysis of the sequences and structures of HIV-1 and HIV-2 proteases. Proteins 10, 325-339.

7 Louis, J.M., et al. (2007) HIV-1 protease: Structure dynamics, and inhibition. $A d v$. Pharmacol. 55, 261-298.

8 Menéndez-Arias, L. (2002) Molecular basis of fidelity of DNA synthesis and nucleotide specificity of retroviral reverse transcriptases. Progr. Nucleic Acid Res. Mol. Biol. 71, 91147.

9 Colson, P., et al. (2004) Polymorphism and drug selected mutations in the protease gene of human immunodeficiency virus type 2 from patients living in Southern France. J. Clin. Microbiol. 42, 570-577.

10 Pieniazek, D., et al. (2004) HIV-2 protease sequences of subtypes A and B harbor multiple mutations associated with protease inhibitor resistance in HIV-1. AIDS 18, 495-502.

11 Damond, F., et al. (2005) Polymorphism of the human immunodeficiency virus type 2 (HIV-2) protease gene and selection of drug resistance mutations in HIV-2-infected patients treated with protease inhibitors. J. Clin. Microbiol. 43, 484-487.

12 Parreira, R., et al. (2006) Natural polymorphisms of HIV type 2 pol sequences from drug naive individuals. AIDS Res. Hum. Retroviruses 22, 1178-1182.

13 Ntemgwa, M., et al. (2007) Natural polymorphisms in the human immunodeficiency virus type 2 protease can accelerate time to development of resistance to protease inhibitors. Antimicrob. Agents Chemother. 51, 604-610.

14 Ruelle, J., et al. (2007) Genetic polymorphisms and resistance mutations of HIV type 2 in antiretroviral-naïve patients in Burkina Faso. AIDS Res. Hum. Retroviruses 23, 955-964. 
15 Ceccherini-Silberstein, F., et al. (2004) Identification of the minimal conserved structure of HIV-1 protease in the presence and absence of drug pressure. AIDS 18, F11-F19.

16 Tözsér, J. and Oroszlan, S. (2003) Proteolytic events of HIV-1 replication as targets for therapeutic intervention. Curr. Pharm. Des. 9, 1803-1815.

17 Tözsér, J., et al. (1991) Studies on the role of the S4 substrate binding site of HIV proteinases. FEBS Lett. 279, 356-360.

18 Tözsér, J., et al. (1992) Kinetic and modeling studies of S3-S3' subsites of HIV proteinases. Biochemistry 31, 4793-4800.

19 Ghosh, A.K., et al. (2006) Structure-based design of novel HIV-1 protease inhibitors to combat drug resistance. J. Med. Chem. 49, 5252-5261.

20 King, N.M., et al. (2004) Combating susceptibility to drug resistance: lessons from HIV-1 protease. Chem. Biol. 10, 1333-1338.

21 Menéndez-Arias, L. (2002) Targeting HIV: Antiretroviral therapy and development of drug resistance. Trends Pharmacol. Sci. 23, 381-388.

22 Johnson, V.A., et al. (2007) Update of the drug resistance mutations in HIV-1: 2007. Top. HIV Med. 15, 119-125.

23 Yoshimura, K., et al. (2002) A potent human immunodeficiency virus type 1 protease inhibitor, UIC-94003 (TMC-126), and selection of a novel (A28S) mutation in the protease active site. J. Virol. 76, 1349-1358.

24 Koh, Y., et al. (2003) Novel bis-tetrahydrofuranylurethane-containing nonpeptidic protease inhibitor (PI) UIC-94017 (TMC114) with potent activity against multi-PI-resistant human immunodeficiency virus in vitro. Antimicrob. Agents Chemother. 47, 3123-3129. 
25 Descamps, D., et al. (2006) In vitro phenotypic susceptibility of HIV-2 clinical isolates to protease inhibitors: amprenavir, atazanavir, lopinavir, and tipranavir. Antivir. Ther. 11, S103.

26 Desbois, D., et al. (2007) Phenotypic susceptibility in vitro to amprenavir, atazanavir, darunavir, lopinavir, and tipranavir of HIV-2 clinical isolates from the French ANRS HIV2 Cohort. $14^{\text {th }}$ Conference on Retroviruses and Opportunistic Infections, Los Angeles, CA, U.S.A., Abstract no. 615.

27 Gong, Y.-F., et al. (2006) Atazanavir susceptibility spectrum extends to HIV-1 non-B subtypes and HIV-2 viral isolates. Antivir. Ther. 11, S100.

28 Rodés, B., et al. (2006) Susceptibility to protease inhibitors in HIV-2 primary isolates from patients failing antiretroviral therapy. J. Antimicrob. Chemother. 57, 709-713.

29 Doyon, L., et al. (2005) Selection and characterization of HIV-1 showing reduced susceptibility to the non-peptidic protease inhibitor tipranavir. Antivir. Res. 68, 27-35.

30 Patick, A.K., et al. (1996) Antiviral and resistance studies of AG1343, an orally bioavailable inhibitor of human immunodeficiency virus protease. Antimicrob. Agents Chemother. 40, 292-297.

31 Masse, S., et al. (2007) In vitro selection and characterization of human immunodeficiency virus type 2 with decreased susceptibility to lopinavir. Antimicrob. Agents Chemother. 51, 3075-3080.

32 Rodés, B., et al. (2006) High rate of proV47A selection in HIV-2 patients failing lopinavirbased HAART. AIDS 20, 127-129.

33 Carrillo, A., et al. (1998) In vitro selection and characterization of human immunodeficiency virus type 1 variants with increased resistance to ABT-378, a novel protease inhibitor. J. Virol. 72, 7532-7241. 
34 Mo, H., et al. (2005) Selection of resistance in protease inhibitor-experienced, human immunodeficiency virus type 1-infected subjects failing lopinavir- and ritonavir-based therapy: mutation patterns and baseline correlates. J. Virol. 79, 3329-3338.

35 Condra, J.H., et al. (1996) Genetic correlates of in vivo viral resistance to indinavir, a human immunodeficiency virus type 1 protease inhibitor. J. Virol. 70, 8270-8276.

36 Molla, A., et al. (1996) Ordered accumulation of mutations in HIV protease confers resistance to ritonavir. Nat. Med. 2, 760-766.

37 Patick, A.K., et al. (1998) Genotypic and phenotypic characterization of human immunodeficiency virus type 1 variants isolated from patients treated with the protease inhibitor nelfinavir. Antimicrob. Agents Chemother. 42, 2637-2644.

38 Masquelier, B., et al. (2002) Human immunodeficiency virus type 1 genotypic and pharmacokinetic determinants of the virological response to lopinavir-ritonavir-containing therapy in protease inhibitor-experienced patients. Antimicrob. Agents Chemother. 46, 2926-2932.

39 Rodés, B., et al. (2000) Emergence of drug resistance mutations in human immunodeficiency virus type 2-infected subjects undergoing antiretroviral therapy. J. Clin. Microbiol. 38, 1370-1374.

40 Van der Ende, M.E., et al. (2003) Clinical, immunological and virological response to different antiretroviral regimens in a cohort of HIV-2-infected patients. AIDS 17 (suppl. 3), S55-S61.

41 Cavaco Silva, J., et al. (2007) Mutations in HIV-2 protease selected by protease-inhibitor therapy. Antivir. Ther. 12, S87.

42 Grossman, Z., et al. (2001) Genotypic variation of HIV-1 reverse transcriptase and protease: comparative analysis of clade C and clade B. AIDS 15, 1453-1460. 
43 Brandin, E., et al. (2003) pol gene sequence variation in Swedish HIV-2 patients failing antiretroviral therapy. AIDS Res. Hum. Retroviruses 19, 543-550.

\section{LEGENDS TO FIGURES}

Figure 1. Targets for therapeutic intervention in the HIV life cycle. Currently approved antiretroviral drugs block HIV infection at different steps of the viral life cycle: (i) virus entry through their interaction with gp120 or gp41(i.e. maraviroc and enfuvirtide, respectively); (ii) reverse transcription (i.e. RT inhibitors); (iii) integration (i.e. integrase (IN) inhibitors such as raltegravir), and (iv) maturation (i.e. PR inhibitors that block the conversion of immature virus into mature infectious virions).

Figure 2. Amino acid sequence comparison of HIV-1 and HIV-2 proteases of reference strains HXB2 (HIV-1) and ROD (HIV-2). Identical residues are marked with red asterisks. Conserved structurally important regions are highlighted on a grey background. Box A corresponds to the dimerization domain, box $\mathrm{B}$ to the active site region and the $\mathrm{C}$-terminal triad, and box $\mathrm{C}$ indicates the flap region. Amino acid differences between HIV-1 and HIV-2 PRs at positions relevant for resistance to HIV-1 PR inhibitors are indicated [22]. Major and minor PR inhibitor resistance mutations occur at the positions highlighted in yellow and light blue, respectively. Highly polymorphic positions ( $\geq 20 \%$ sequence variation) in the PR of HIV-2 subtype A isolates are indicated with blue letters. HIV-2 PR residues appearing with a frequency above 4 $\%$ are indicated below the reference sequence. The analysis of polymorphic sites in the HIV-2 PR was based on 164 published sequences of HIV-2 isolates obtained from naïve patients [9$14]$. 
Figure 3. Crystallographic structures of human immunodeficiency virus proteases complexed with indinavir. In panels (A) and (B), the two polypeptide chains of the human immunodeficiency virus type 2 protease are represented as ribbon diagrams (red and green). The inhibitor is shown in yellow using a CPK model. The side chains chains of catalytic residues (Asp-25 in both subunits) are shown in blue. Atom coordinates were taken from PDB file 1HSH. Side and top views are shown in (A) and (B), respectively. (C) Crystal structure of human immunodeficiency virus type 1 protease complexed with indinavir (PDB file 1HSG).

Figure 4. Residues forming the substrate binding pocket of HIV proteases. Schematic representation of an oligopeptide (SQNY $\downarrow$ PIV) that mimicks the HIV-1 Gag MA $\downarrow$ CA cleavage site, showing its potential interactions with the substrate binding site of the HIV-2 protease. Primes indicate residues in the second subunit of the dimer. The relative size of each substrate is illustrated by the area enclosed by the curved line around each substrate side chain. Substrate binding residues that are commonly observed in HIV isolates with high-level resistance to one or more drugs are boxed, and the residue found in the wild-type HIV-1 is shown between parentheses. The amino acid residues interacting with ritonavir, lopinavir and darunavir (inhibitors shown in white) in the crystal structures of HIV-1 PR complexes are indicated below. Sequence positions of residues at less than $4 \AA$ from the inhibitor are given. Side chains and their corresponding amino acid are indicated for those residues located at less than $3.5 \AA$ of the inhibitor.

Figure 5. Inhibitors of human immunodeficiency virus type 1 (HIV-1) protease that are licensed to treat AIDS and AIDS-related malignancies. 